\title{
Cognition, sleep and respiration in at-risk children treated for obstructive sleep
} apnoea

\author{
H.E. Montgomery-Downs, V.M. Crabtree and D. Gozal
}

ABSTRACT: Sleep-disordered breathing in children has been associated with cognitive impairment. The purpose of this study was to examine the impact of tonsillectomy and adenoidectomy (T\&A) on sleep, respiration and cognitive function in children of pre-school age with obstructive sleep apnoea (OSA) from a low-income community population.

Altogether, 19 children attending state-funded pre-school programmes underwent overnight polysomnography and cognitive assessment before and following surgical treatment for OSA; 19 matched controls were also assessed.

Following T\&A, OSA subjects' delta sleep increased, rapid eye movement sleep decreased, and respiratory and arousal indices improved. There were no significant differences in OSA subjects' post-operative sleep or respiratory measures compared to controls. Prior to T\&A, cognitive scores were significantly lower in OSA subjects versus controls; following T\&A, OSA subjects' scores improved compared to pre-operative scores and did not differ from those of matched controls.

Following tonsillectomy and adenoidectomy, at-risk pre-schoolers recruited directly from the community showed normalised sleep and respiratory patterns and improved cognitive scores. These findings, in this uniquely vulnerable population, which is unlikely to seek evaluation and treatment for obstructive sleep apnoea, underscore the potential value of outreach screening programmes for sleep-disordered breathing, particularly among low-income groups of pre-school age.

KEYWORDS: Cognition, development, obstructive sleep apnoea, respiration, sleep

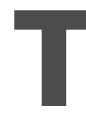
he prevalence of habitual snoring in otherwise healthy children varies geographically and has been reported to be 6$27 \%$ [1-3]. Snoring is the cardinal symptom of sleep-disordered breathing (SDB), which encompasses obstructive sleep apnoea (OSA) and upper airway resistance syndrome [4]. SDB may be present in up to $3 \%$ of children and is associated with sleep fragmentation and gas exchange abnormalities. Children with SDB are more frequent users of healthcare services [5], experience more frequent comorbid chronic illnesses [1, 6], may develop significant clinical cardiovascular morbidity [7] and also display daytime behavioural manifestations, including attention deficit/hyperactivity disorder [8, 9], as well as other psychiatric and behavioural comorbid conditions [10-12]. In addition to cardiovascular and behavioural complications, SDB in children has been associated with impairment of normal

For editorial comments see page 216. cognitive development $[8,13]$ and school performance [2, 14, 15].

The preferred treatment for OSA in otherwise healthy children is tonsillectomy and adenoidectomy (T\&A) [16, 17]. Although T\&A may not be universally effective in paediatric patients [18], several studies have shown that post-operative recovery from OSA treated with T\&A includes normalisation of sleep and respiratory measures $[16,17,19-21]$, and improvements in school performance [2], cognitive function [13, 22, 23], behaviour [24] and quality of life [25, 26].

Thus, the cumulative evidence indicates that paediatric SDB is a frequent condition leading to significant clinical morbidity and that treatment for OSA in paediatric populations leads to improvements in clinical outcome, including cognitive function. Taken together, these studies support the need for early intervention. However, most studies on the reversibility of cognitive function deficits have been restricted to

\section{AFFILIATIONS}

Division of Pediatric Sleep Medicine, Dept of Pediatrics, University of Louisville, Louisville, KY, USA.

\section{CORRESPONDENCE}

D. Gozal

Kosair Children's Hospital Research Institute

Dept of Pediatrics

570 South Preston Street

Suite 321

Louisville

KY 40202

USA

Fax: 15028522215

E-mail: david.gozal@louisville.edu

Received:

July 092004

Accepted after revision:

September 282004

SUPPORT STATEMENT

This work was supported, in part, by US Department of Education (Washington, DC, USA) grant H324E011001, Centers for Disease Control and Prevention (Atlanta, GA, USA) grant E11/CCE 422081-01, and National Institutes of Health (Bethesda, MD, USA) grants HL62570 and F32 HL-074591. 
small clinically referred populations encompassing a broad age range. The goal of the present study was to examine the impact of T\&A in young children with OSA recruited from a highly vulnerable at-risk community population, who had not previously sought medical evaluation for the potential presence of SDB.

\section{METHODS}

The study was approved by the Institutional Review Boards of the University of Louisville and Norton Healthcare (both Louisville, KY, USA); informed consent was obtained separately for participation in the parental report questionnaire and overnight polysomnography (PSG) elements of the study.

\section{Questionnaire instrument}

The information collected included the demographics of both parents and the child, and child behaviours, including frequency and loudness of snoring. Report options for snoring frequency included: never (never in the past 6 months), rarely (once a week), occasionally (twice weekly), frequently (three or four times a week) and almost always (more than four times a week). Subjects whose parents indicated that snoring occurred occasionally or more frequently were contacted by telephone and invited to participate in the second phase of the study, including overnight PSG and cognitive assessment. This instrument has previously demonstrated a high sensitivity when used in the same population [27].

\section{Participants}

Subjects attended state-sponsored Early Jump Start pre-school programmes, admission to which is limited to children who are disadvantaged either developmentally, as determined through an Individual Education Plan, or financially. Families fulfilling the latter criterion must be financially eligible for the National School Lunch Program. For a family of four, the maximum gross annual household income for eligibility during the initial year of the study was US\$22,945; the national poverty level during this period was $\$ 18,400$. During the academic years studied, $91 \%$ of children were admitted to Early Jump Start on the basis of family income eligibility (D. Corson, Assessment, Research, and Planning, Jefferson County Public Schools, Louisville, KY, USA, personal communication). Questionnaires were sent home with all children enrolled in Early Jump Start pre-school classes, to be completed by the parent(s) and returned to school, where they were collected by a researcher. The subjects who were invited to participate further in the study were those admitted to Early Jump Start programmes based upon financial eligibility; survey participants who were developmentally disadvantaged or who had overt craniofacial abnormalities and whose survey indicated risk for SDB were referred to the affiliated sleep medicine clinic.

Qualifying subjects were recruited for overnight PSG assessment based on parental reported questionnaires previously validated using the same population [27]. Subjects who were subsequently diagnosed with OSA and who underwent T\&A were invited to return for follow-up assessments.

\section{Control subjects}

Classmates from the same socio-economically at-risk population whose survey results indicated that they had no history of snoring were matched to the OSA subjects on the basis of age (median of the OSA subjects' pre- and post-operative assessment ages), as well as sex, ethnicity and highest level of maternal education achieved (table 1). These matched controls underwent PSG and cognitive testing on a single occasion. Repeat testing was not administered to the matched healthy controls.

\section{Overnight polysomnography}

Subjects were excluded from this phase of the investigation on the basis of incomplete questionnaires and/or contact information, chronic medical conditions, and genetic or overt craniofacial abnormalities, and/or when they had already sought medical evaluation for snoring. No PSG was performed on a night on which a child was acutely ill.

A standard overnight multichannel PSG evaluation was performed in the Sleep Medicine Center at Kosair Children's Hospital (Louisville, KY, USA). Children were studied for up to $12 \mathrm{~h}$ in a quiet darkened room with an ambient temperature of $\sim 24^{\circ} \mathrm{C}$ with a parent or guardian present. Lights out was 21:32 h (SD $19.1 \mathrm{~min}$ ) and lights on was 06:20 h (SD $13.0 \mathrm{~min}$ ). No medications were used to induce sleep.

The following parameters were measured: chest and abdominal wall movement by respiratory impedance or inductance plethysmography; cardiac frequency by ECG; and airflow with a side-stream end-tidal capnograph (BCI SC-300; Redding Medical, Inc., Finksburg, MD, USA), which also provided breath-by-breath assessment of end-tidal carbon dioxide tension $\left(P \mathrm{ET}, \mathrm{CO}_{2}\right)$ and nasal pressure, and an oral-nasal airflow thermistor. Arterial oxygen saturation measured by pulse oximetry $\left(\mathrm{Sp}_{\mathrm{p}} \mathrm{O}_{2}\right)$ was assessed (Nellcor $\mathrm{N}$ 100; Nellcor, Inc. Hayward, CA, USA), with a simultaneously recorded pulse waveform. A bilateral electro-oculogram, eight channels of

\begin{tabular}{|c|c|c|c|}
\hline \multirow[t]{2}{*}{ TABLE 1} & \multicolumn{3}{|c|}{$\begin{array}{l}\text { s and maternal characteristics of } \\
\text { e and after adenoidectomy and } \\
\text { and of matched controls }\end{array}$} \\
\hline & Before & After & Control \\
\hline Subjects $\mathbf{n}$ & 19 & $19^{\#}$ & $19^{\circ}$ \\
\hline \multicolumn{4}{|l|}{ Age yrs } \\
\hline Questionnaire completion & $4.2 \pm 0.77$ & $4.2 \pm 0.77^{\#}$ & $4.3 \pm 0.67$ \\
\hline PSG & $4.4 \pm 0.70$ & $4.9 \pm 0.70$ & $4.5 \pm 0.64$ \\
\hline Males \% & 53 & $53^{\#}$ & $53^{\bullet}$ \\
\hline \multicolumn{4}{|l|}{ Ethnicity \% } \\
\hline Caucasian & 32 & $32^{\#}$ & $32^{\circ}$ \\
\hline African-American & 58 & $58^{\#}$ & $58^{\circ}$ \\
\hline Asian/Pac. I. or other & 10 & $10^{\#}$ & $10^{\circ}$ \\
\hline \multicolumn{4}{|l|}{ Maternal education \% } \\
\hline Junior high school & 5 & $5^{\#}$ & $5^{\circ}$ \\
\hline High school & 68 & $68^{\#}$ & $68^{\circ}$ \\
\hline College & 21 & $21^{\#}$ & $21^{\bullet}$ \\
\hline Graduate school & 5 & $5^{\#}$ & $5^{\bullet}$ \\
\hline $\begin{array}{l}\text { Data are presented as mea } \\
\text { Pac. I.: Pacific Islander. } \# \text { : } \\
\text { matched on this variable: no }\end{array}$ & $\begin{array}{l}\text { or percer } \\
\text { unchang } \\
\text { ence. }\end{array}$ & $\begin{array}{l}\text { SSG: pol } \\
\text { ost-operative }\end{array}$ & $\begin{array}{l}\text { mnography; } \\
\because \text { controls }\end{array}$ \\
\hline
\end{tabular}


electroencephalogram, chin and anterior tibial electromyograms, and analogue output from a body position sensor (Braebon Medical Corporation, Ogdensburg, NY, USA) were also monitored. All measures were digitised using a commercially available PSG system (MedCare Diagnostics, Buffalo, NY, USA). Tracheal sound was monitored with a microphone sensor (Sleepmate; Rochester Electro-Medical, Inc., Tampa, FL, USA) and digital synchronised video images were collected.

\section{Sleep and respiratory characteristics}

Sleep architecture was assessed using standard techniques [28]. Central, obstructive and mixed apnoeic events were scored using changes measured with an oral-nasal thermistor and/or end-tidal carbon dioxide cannula. Obstructive apnoea was defined as the absence of airflow with continued chest wall and abdominal movement for at least two breaths $[29,30]$. Hypopnoeas were defined as a decrease in nasal flow of $\geqslant 50 \%$ with a corresponding decrease in $\mathrm{Sp}, \mathrm{O}_{2}$ of $\geqslant 4 \%$ and/or arousal [30]. The apnoea/hypopnoea index (AHI) was defined as the number of apnoeas and hypopnoeas per hour of total sleep time (TST). For treatment referral purposes, only patients with an obstructive AHI of $>5$ events $\cdot \mathrm{h}^{-1}$ were considered as candidates for T\&A and referred for surgical intervention. Mean $S_{\mathrm{p}, \mathrm{O}_{2}}$ and $\mathrm{Sp}_{\mathrm{p}} \mathrm{O}_{2}$ nadir were determined. The mean and peak $P \mathrm{ET}, \mathrm{CO}_{2}$ were determined. Since criteria for arousal have not yet been developed for children [31], arousals were defined as recommended by the American Sleep Disorders Association Task Force report [32], and included respiratory-related (occurring immediately subsequent to an apnoea, hypopnoea or snore), technician-induced and spontaneous arousals. Respiratory arousal index was expressed as the total number of respiratoryrelated arousals per hour of TST; total arousal index was expressed as the number of combined arousals per hour of TST.

\section{Cognitive assessments}

At 08:00 $\mathrm{h}$ on the morning following PSG and lasting $\sim 45 \mathrm{~min}$, developmentally appropriate cognitive assessments were administered. The Differential Ability Scales (DAS) [33], a measure of general conceptual ability (GCA), were administered. The pre-school version is intended for use with children between 2 yrs 6 months and 5 yrs 11 months of age, and has attractive graphics that are appealing and engaging for preschool children. Assessments of verbal (verbal comprehension and naming vocabulary) and nonverbal ability (picture similarities, pattern construction, copying and early number concepts) and block building were administered. Scores are reported as standard scores with a mean \pm SD of $100 \pm 15$.

The Pre-Reading Abilities subtests from the Developmental Neuropsychological Assessment (NEPSY) [34] were used to obtain measures of phonological processing, sentence comprehension and naming. This scale has been standardised for use with children aged 3-12 yrs. Scores are reported as subtest scaled scores with a mean \pm SD of $10 \pm 3$.

Examiners were blinded to subjects' diagnostic status and different reliable examiners performed post-operative assessments.

\section{Statistical analyses}

Repeated-measures ANOVA (general linear model) was used to test for significant differences between pre- and posttreatment sleep, respiratory and cognitive measures, as well as between OSA subjects' pre- and post-treatment measures compared to matched controls. A p-value of $\leqslant 0.05$ was considered significant. With 19 subjects and a difference in SD of 0.5 between DAS scores significant when one-tailed $\alpha$ was 0.05 , the statistical power of the study was 0.83 .

\section{RESULTS}

\section{Characteristics of sample population}

There were 1,951 respondents to the questionnaire during the combined 2001 and 2002 academic years, which represented a response rate of $32.8 \%$. The ethnic distribution of those who responded to the survey (table 1) was similar to that of the general pre-school population during the same academic years (D. Corson, personal communication).

Between October 2001 and June 2003, 273 pre-schoolers underwent PSG and cognitive testing. Of these, 39 were diagnosed as having SDB, and it was recommended by the medical director of the study that the family discuss referral for surgical treatment with the child's primary care physician. Of these 39, nine families could not be contacted to determine treatment status despite repeated attempts, five had not followed treatment recommendations within 13 months and the remaining 25 were known to have undergone T\&A. Of the 25 who received treatment, five repeatedly did not attend follow-up appointments, and one refused to participate a second time (fig. 1). Thus 19 children underwent T\&A (between 23 days and 8.1 months following initial PSG (mean \pm SD $2.9 \pm 2.1$ months)) and returned for post-operative evaluation $2.9-6.3$ months $(4.0 \pm 1.0$ months) following surgery, completing all phases of the study, and were therefore available for analysis (table 1). The time from initial PSG and cognitive assessment to the follow-up for these OSA subjects was $6.9 \pm 2.3$ months.

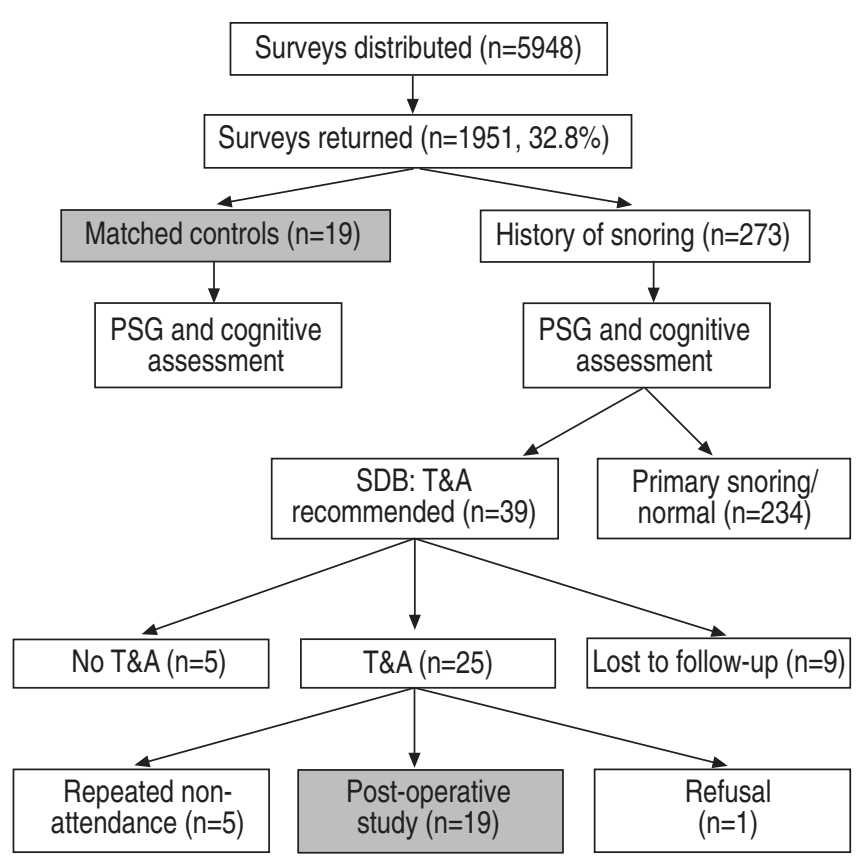

FIGURE 1. Subject and matched control recruitment. PSG: polysomnography: SDB: sleep-disordered breathing; T\&A: tonsillectomy and adenoidectomy 


\section{Sleep architecture and respiratory measures}

Following T\&A, the percentage of delta sleep increased and that of rapid eye movement (REM) sleep decreased in OSA subjects. Compared to controls, the pre-operative percentage of time awake and of delta sleep were reduced. Following T\&A, respiratory arousal index, total arousal index, AHI and apnoea index were all improved. There were no significant differences between OSA post-operative sleep or respiratory measures and those of controls (table 2).

\section{Cognitive function}

Prior to T\&A, DAS scores were significantly lower in OSA subjects than in controls (table 3). Following T\&A, OSA DAS scores improved significantly compared to pre-operative scores and did not differ from those of the matched controls (table 3). Sixteen of the 19 OSA subjects improved their GCA scores post-operatively by one to 22 points (mean \pm SEM $6.9 \pm 1.4)$ The scores of three OSA subjects decreased postoperatively by five (two) to nine (one) points.

Cognitive assessments were administered to all subjects. DAS GCA scores were obtained for all; six pre-operative, one postoperative and five control administrations resulted in GCA being pro-rated due to a single missing subtest. Thus the data for individual verbal and nonverbal subtests were not compared for post-operative improvement analysis due to insufficient statistical power.

Since this population of children was very difficult to assess due to their young age and developmentally at-risk status, NEPSY subtest results were sometimes unavailable for analysis due to administration difficulties. As a result, data on only 11, 15 and 18 subjects were available for pre-/post-operative comparisons for phonological processing, sentence repetition and verbal fluency, respectively. NEPSY scores were not found to change or differ from controls on any measure except for verbal fluency (OSA subject scores were consistently lower than those of controls both before and after T\&A).

\section{DISCUSSION}

The present at-risk population cohort of children with OSA, who were matched to a control group for median age between the initial and follow-up assessment, sex, ethnicity and maternal education, showed normalisation of their sleep and breathing patterns and improved cognitive scores following surgical treatment. The current sample was recruited directly from the community and represents a unique and especially vulnerable population that is ordinarily unlikely to seek evaluation and treatment for SDB. The current study underscores the potential value of outreach screening programmes for SDB, particularly within low-income groups of pre-school age.

The OSA subjects in the present community-recruited study showed sleep architecture improvements post-operatively; namely, their latency to sleep onset decreased and the percentage of time spent in delta sleep increased. These findings are inconsistent with the only previously reported clinical trial reporting sleep stage percentages before and after $\mathrm{T} \& \mathrm{~A}$, in which no significant changes in overall sleep stage percentages were found [21]. Since a developmental trajectory exists for changes in sleep stage percentage distribution throughout the night, the present authors suspect that, although the number of subjects included in the present study was relatively low, the narrow age range included in the present cohort is likely to have afforded greater statistical

TABLE 2 Polysomnographic characteristics of children with sleep-disordered breathing before and after tonsillectomy and adenoidectomy, and of matched controls

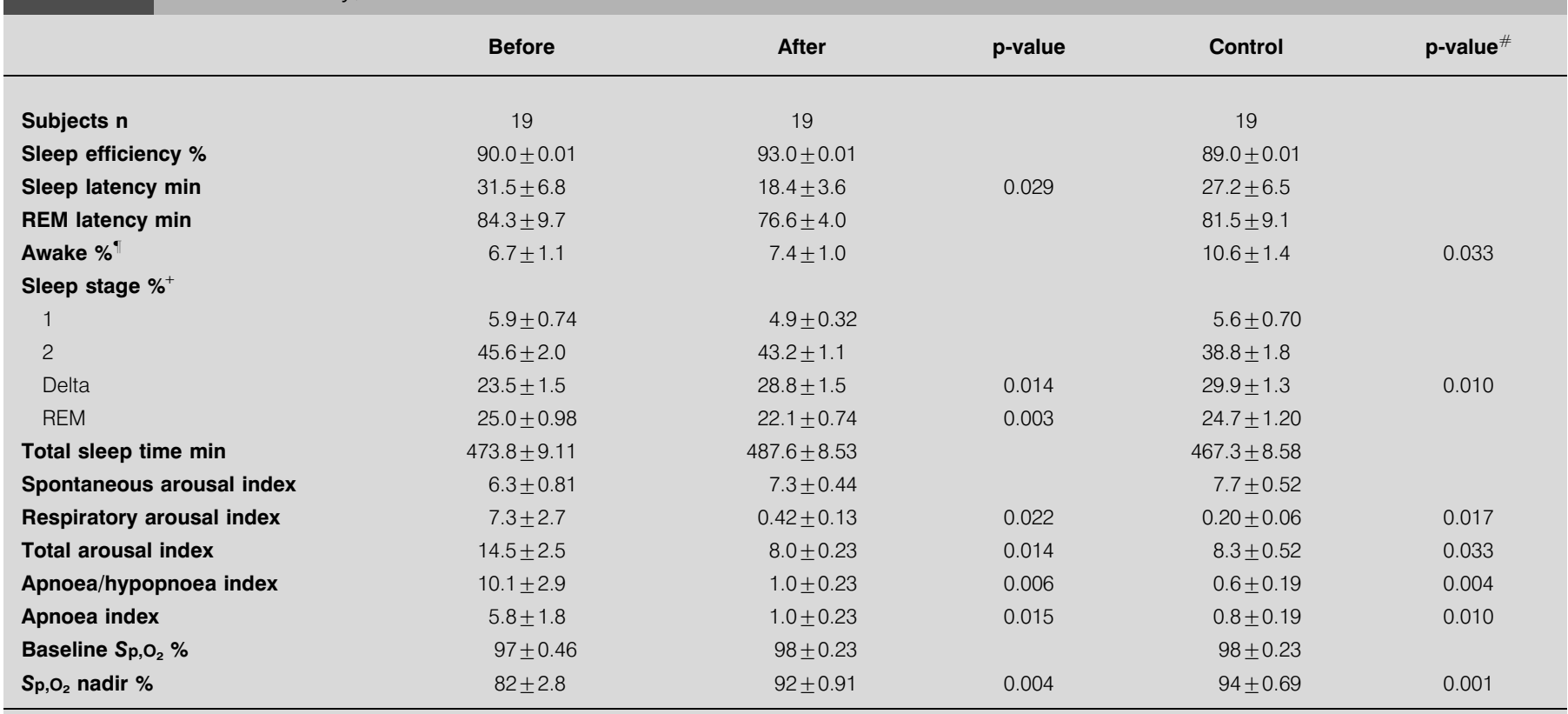

Data are presented as mean \pm SEM. REM: rapid eye movement; $\mathrm{Sp}, \mathrm{O}_{2}$ : arterial oxygen saturation measured by pulse oximetry. ${ }^{\#}:$ control versus pre-operative value; ${ }^{\bullet}$ calculation based on time from lights out to lights on; ${ }^{+}$: calculation based on total sleep time. 


\begin{tabular}{|c|c|c|c|c|c|c|}
\hline \multirow[t]{3}{*}{ TABLE 3} & $\begin{array}{l}\text { Differential Abilit } \\
\text { tonsillectomy, a }\end{array}$ & $\begin{array}{l}\text { erformance i } \\
\text { eed controls }\end{array}$ & with sleer & d breathing & afte & ctomy and \\
\hline & \multirow[t]{2}{*}{ Before } & \multirow[t]{2}{*}{ After } & \multirow[t]{2}{*}{ p-value ${ }^{\#}$} & \multirow[t]{2}{*}{ Control } & \multicolumn{2}{|c|}{ p-value ${ }^{\#}$} \\
\hline & & & & & $1^{\prime}$ & $2^{+}$ \\
\hline Score $^{\S}$ & $82.52 \pm 14.03$ & $87.16 \pm 14.65$ & 0.02 & $93.95 \pm 24.93$ & 0.01 & 0.13 \\
\hline
\end{tabular}

power, and therefore permitted identification of significant improvements in sleep architecture after T\&A.

An alternative possibility could include a greater propensity for SDB-induced disruption of sleep architecture in socio-economically at-risk paediatric populations, such that surgical correction of SDB could result in increased differences between pre- and post-operative sleep characteristics. Notwithstanding such considerations, when the post-operative sleep measures of OSA subjects were compared with those of controls matched for socio-economic factors, a complete normalisation of sleep measures was found, to the extent that there were no longer significant differences from matched controls.

The decrease in the percentage of REM sleep following treatment for OSA in the present study was unexpected and in conflict with previously published data showing that an increased percentage of REM sleep is associated with improvements in both DAS and NEPSY scores in a slightly older broad community sample [8]. However, the percentage of REM sleep of OSA subjects did not differ from that of control subjects pre- or post-operatively. Although significant, the percentage difference between pre- and post-operative percentages of REM sleep in the current study translates to a relatively low mean difference in absolute duration of REM sleep (3.4 min). Regardless, this discrepancy emphasises the need for more intensive study of the relationship between sleep and neurocognitive function in young children who are at-risk and otherwise.

Arousal measure indices also normalised among the at-risk children in the OSA group after surgery. Indeed, the total arousal index was almost halved post-operatively; this was accounted for by the almost complete abrogation of respiratory arousals. In contrast, the spontaneous arousal index did not differ post-operatively, and nor did values for either the pre- or post-operative measures differ from those of matched controls. Despite the high variability in the scoring criterion for arousal among different centres, the post-operative reduction in the respiratory arousal index in the present study was consistent with previous findings from available clinical trials $[17,21]$.

As expected, and previously reported [16, 17, 19-21], marked pre-to-post-surgical changes in respiratory indices during sleep were found. Both the AHI and apnoea index were reduced after T\&A, and no longer differed from those of matched controls. These findings further corroborate the relatively high short-term efficacy of T\&A in paediatric OSA [18]. Blood oxygenation in the absence of any respiratory events (baseline $\mathrm{S}, \mathrm{O}_{2}$ ) did not differ pre- and post-operatively or compared to controls subjects; $\mathrm{Sp}_{\mathrm{p}} \mathrm{O}_{2}$ nadir improved pre-to-post-surgically, was lower prior to surgery compared to controls and did not differ from controls following surgery.

Concurrent with changes in sleep, arousal and respiratory measures, improvements in cognitive function measures were identified, with normalisation of complex mental functions after T\&A compared to matched controls, as measured by the DAS. These findings are particularly encouraging since children in the current sample were already at high risk of reduced capacity in performing complex mental functions due to their low socio-economic status and environmental impoverishment. Both the sleep fragmentation and abnormal blood gas exchange that ensue from upper airway obstruction during sleep in OSA may induce substantial alterations in brain function and structure in general [35], and, more specifically, affect the prefrontal cortex, leading to impairments in cognitive and executive functional processing [36]. However, the present study suggests that these cognitive impairments may be reversible in very young children upon efficacious treatment.

As mentioned above, there is a paucity of information on the reversibility of cognitive morbidity associated with paediatric SDB. However, animal models have revealed long-term partially irreversible impairments in learning, memory and executive function, when the intermittent hypoxia that characteristically accompanies SDB occurs early in development [36-40]. Interestingly, environmental enrichment reduces the susceptibility to cognitive dysfunction following exposures to intermittent hypoxia during sleep [41]. It could be argued that environmental impoverishment would enhance the deleterious effects of SDB. Indeed, in the present study, the mean DAS scores for OSA subjects' post-operative assessments remain below the standard means, as do those of the matched control subjects. Thus the present authors submit that socio-economically challenged paediatric populations who do not have access to environments that are conducive to optimal learning and brain development opportunities prior to the onset of the intermittent hypoxia associated with OSA may be particularly susceptible to the deleterious effects of this disorder.

\section{Limitations}

There are several potential limitations to the present study. Attempts were made to administer components of the NEPSY. However, administration of a relatively large battery of tests to such young children is fraught with substantial difficulties, particularly as regards achieving the necessary sustained attention and motivation to successfully and reliably complete such tests. Thus several of the children enrolled in this study were unable to complete the last few items of the NEPSY, 
leading to insufficient statistical power in the comparative analyses of these subtests. Future studies assessing similar populations may benefit from a school-based assessment approach in which testing is administered over a series of days rather than in a single session.

The assessments used are designed to be stable over time, but, because the OSA subjects were tested twice, improvements due to practice effects should be considered. The DAS manual states that "we must keep practice effects in mind when interpreting the scores of a child retested within a few months of initial testing" (p. 184) and further adds that GCA has been shown to vary by approximately three points due to practice effects [33]. Since OSA subjects' initial and follow-up assessments were separated by $6.9 \pm 2.3$ months (mean \pm SD), this was sufficient to nullify this concern.

A related limitation was that, among this at-risk population, it proved difficult to find families willing to participate as control subjects and impossible to expect them to return for a followup assessment. As such, cognitive assessments were administered to the control subjects on a single occasion, whereas the OSA subjects were tested twice. Since all subjects were enrolled in a pre-school programme designed to provide an enriching academic experience, it is possible that both OSA and control subjects alike may have shown improvements in their cognitive scores over time. In view of this potential confounder, controls were matched to the intermediary age between the pre- and post-operative assessments of the OSA subjects, such that the groups could be compared since the beneficial effects of the academic programme would apply to both OSA and control subjects.

It is possible that the subjects participating in the present study were vulnerable to first-night effects, and that these may have masked post-operative changes in sleep efficiency, a particular concern when the number of subjects is relatively low. However, all children in both the OSA and control groups were exposed to the same environmental conditions. Furthermore, a recent study reported that children with OSA did not experience first-night effects differently from other 2-6yr-old children, and that respiratory parameters were not vulnerable to the presence of first-night effects [42].

Although all the children were sampled from an at-risk population, the subjects whose participation was complete, i.e. those who filled in and returned the screening questionnaire, participated in initial PSG evaluation and cognitive assessment, followed treatment recommendations and returned for postoperative evaluation, could differ from those who selected not to participate in one or all of the phases of the study. It is possible that this self-selection bias is somewhat alleviated by the intraindividual pre-/post-treatment comparisons, and by the fact that such biases are also applicable to the control population.

It is also possible that adult illiteracy may have played a role in the low response rates to the survey questionnaire, that difficulty with transportation and inconsistent housing may have prevented some families from participating in the initial or follow-up evaluations, and that problems with health insurance may have prevented some families from seeking the recommended treatment. Nevertheless, although these concerns are clearly worthy of consideration, it is unlikely that they contributed to the dynamic pre- and post-operative changes in sleep characteristics and cognitive function in the OSA-afflicted children. Overcoming these limitations through studies using assistance-based recruitment and clinically based outreach programmes may confirm and further extend the present findings, and delineate the benefits of such early assessment by providing more accurate estimates regarding the amelioration and extent of recovery from the cognitive consequences associated with OSA.

In summary, the present study has shown the difficulties inherent in screening for sleep-disordered breathing in a low socio-economic status population of children, and further emphasises the importance of such an approach in the context of the present findings, which demonstrate that sleep-disordered breathing is associated with substantial cognitive morbidity among these children. More importantly, treatment of sleepdisordered breathing may lead to normalisation of sleep and reversibility of the cognitive impairment in this population. These encouraging results in a population that would be highly unlikely to seek medical care for sleep-disordered breathing support the institution of a school-based screening programme at this early age in order to prevent long-lasting morbid consequences of sleep-disordered breathing.

\section{ACKNOWLEDGEMENTS}

The authors would like to thank the parents and children who participated in the present study, twice in many cases. J. Bruner, C. Klaus and N. Smith performed the polysomnographic recordings. C. McCliment, N. McNally, T. Raffield and J. Rutherford administered the cognitive assessments. V. Molfese, J. Neamon, J. Walker, S. Berg, J. Berg and B. Brown collaborated on the validation of testing data. C. Holbrook provided logistical support.

\section{REFERENCES}

1 Archbold KH, Pituch KJ, Panahi P, Chervin RD. Symptoms of sleep disturbances among children at two general pediatric clinics. J Pediatr 2002; 140: 97-102.

2 Gozal D. Sleep-disordered breathing and school performance in children. Pediatrics 1998; 102: 616-620.

3 Gislason T, Benediktsdottir B. Snoring, apneic episodes, and nocturnal hypoxemia among children 6 months to 6 years old. An epidemiologic study of lower limit of prevalence. Chest 1995; 107: 963-966.

4 Carroll JL, McColley SA, Marcus CL, Curtis S, Loughlin GM. Inability of clinical history to distinguish primary snoring from obstructive sleep apnea syndrome in children. Chest 1995; 108: 610-618.

5 Reuveni H, Simon T, Tal A, Elhayany A, Tarasiuk A. Health care services utilization in children with obstructive sleep apnea syndrome. Pediatrics 2002; 110: 68-72.

6 Rona JR, Li L, Gulliford MC, Chinn S. Disturbed sleep: effects of sociocultural factors and illness. Arch Dis Child 1998; 78: 20-25.

7 Amin RS, Kimball TR, Bean JA, et al. Left ventricular hypertrophy and abnormal ventricular geometry in children and adolescents with obstructive sleep apnea. Am J Respir Crit Care Med 2002; 165: 1395-1399.

8 O'Brien LM, Holbrook CR, Mervis CB, et al. Sleep and neurobehavioral characteristics of 5-7 year-old children 
with parentally reported symptoms of ADHD. Pediatrics 2003; 111: 554-563.

9 Chervin RD, Dillon JE, Bassetti C, Ganoczy DA, Pituch KJ. Symptoms of sleep disorders, inattention, and hyperactivity in children. Sleep 1997; 20: 1187-1192.

10 Aronen ET, Paavonen EJ, Fjällberg $M$, Soininen $M$, Törrönen J. Sleep and psychiatric symptoms in school-age children. J Am Acad Child Adolesc Psychiatry 2000; 39: 502-508.

11 Lewin DS, Rosen RC, England SJ, Dahl RE. Preliminary evidence of behavioral and cognitive sequelae of obstructive sleep apnea in children. Sleep Med 2002; 3: 5-13.

12 Gottlieb DJ, Vezina RM, Chase C, et al. Symptoms of sleepdisordered breathing in 5-year-old children are associated with sleepiness and problem behaviors. Pediatrics 2003; 112: 870-877.

13 Stradling JR, Thomas G, Warley AR, Williams P, Freeland A. Effect of adenotonsillectomy on nocturnal hypoxemia, sleep disturbance, and symptoms in snoring children. Lancet 1990; 335: 249-253.

14 Montgomery-Downs HE, Jones VF, Molfese VJ, Gozal D. Snoring in preschoolers: potential associations with sleepiness, ethnicity, and learning. Clin Pediatr (Phila) 2003; 42: 719-726.

15 Gozal D, Pope DW. Snoring during early childhood and academic performance at ages 13-14 years. Pediatrics 2001; 107: 1394-1399.

16 Wiet GJ, Bower C, Seibert R, Griebel M. Surgical correction of obstructive sleep apnea in the complicated pediatric patient documented by polysomnography. Int J Paediatr Otorhinolaryngol 1997; 41: 133-143.

17 Nieminen $P$, Tolonen $U$, Löppönen $H$. Snoring and obstructive sleep apnea in children: a 6-month follow-up study. Arch Otolaryngol Head Neck Surg 2000; 126: 481-486.

18 Lipton AJ, Gozal D. Treatment of obstructive sleep apnea in children: do we really know how? Sleep Med Rev 2002; 7: 61-80.

19 Nishimura T, Morishima N, Hasegawa S, Shibata N, Iwanaga K, Yagisawa M. Effect of surgery on obstructive sleep apnea. Acta Otolaryngol 1996; 523: Suppl. 1, 231-233.

20 Jain A, Sahni JK. Polysomnographic studies in children undergoing adenoidectomy and/or tonsillectomy. J Laryngol Otol 2002; 116: 711-715.

21 Tal A, Bar A, Leiberman A, Tarasiuk A. Sleep characteristics following adenotonsillectomy in children with obstructive sleep apnea syndrome. Chest 2003; 124: 948-953.

22 Ali NJ, Pitson D, Stradling JR. Sleep disordered breathing: effects of adenotonsillectomy on behavior and psychological functioning. Eur J Pediatr 1996; 155: 56-62.

23 Friedman BC, Hendeles-Amitai A, Kozminsky E, et al., Adenotonsillectomy improves neurocognitive function in children with obstructive sleep apnea syndrome. Sleep 2003; 26: 999-1005.

24 Goldstein NA, Post C, Rosenfeld RM, Campbell TF. Impact of tonsillectomy and adenoidectomy on child behavior. Arch Otolaryngol Head Neck Surg 1998; 124: 494-498.

25 de Serres LM, Derkay C, Sie K, et al. Impact of adenotonsillectomy on quality of life in children with obstructive sleep disorders. Arch Otolaryngol Head Neck Surg 2002; 128: 489-496.

26 Goldstein NA, Fatima M, Campbell TF, Rosenfeld RM. Child behavior and quality of life before and after tonsillectomy and adenoidectomy. Arch Otolaryngol Head Neck Surg 2002; 128: 770-775.

27 Montgomery-Downs HE, O'Brien LM, Holbrook R, Gozal D. Snoring and sleep-disordered breathing in young children: subjective and objective correlates. Sleep 2004; 27: 87-94.

28 Rechtschaffen A, Kales A. A Manual of Standardized Terminology, Techniques and Scoring System for Sleep Stages of Human Subjects. Los Angeles, CA, Brain Information Services/Brain Research Institute, University of California, 1968.

29 Marcus CL, Omlin KJ, Basinski DJ, et al. Normal polysomnographic values for children and adolescents. Am Rev Respir Dis 1992; 156: 1235-1239.

30 American Thoracic Society. Standards and indications for cardiopulmonary sleep studies in children. Am J Respir Crit Care Med 1996; 153: 866-878.

31 Bandla HPR, Gozal D. Dynamic changes in EEG spectra during obstructive apnea in children. Pediatric Pulmonol 2000; 29: 359-365.

32 Sleep Disorders Atlas Task Force. EEG arousals: scoring and rules and examples: a preliminary report from the Sleep Disorders Atlas Task Force of the American Sleep Disorders Association. Sleep 1992; 15: 173-184.

33 Elliott CD. Differential Ability Scales: Introductory and Technical Handbook. San Antonio, TX, The Psychological Corporation, Harcourt Brace Jovanovich, Inc., 1983.

34 Korkman M, Kirk U, Kemp S. A Developmental Neuropsychological Assessment Manual. San Antonio, TX, The Psychological Corporation, Harcourt Assessment, 1998.

35 Macey PM, Macey KE, Henderson LA, et al. Functional magnetic resonance imaging responses to expiratory loading in obstructive sleep apnea. Respir Physiol Neurobiol 2003; 138: 275-290.

36 Beebe DW, Gozal D. Obstructive sleep apnea and the prefrontal cortex: towards a comprehensive model linking nocturnal upper airway obstruction to daytime cognitive and behavioral deficits. J Sleep Res 2002; 11: 1-16.

37 Gozal E, Row BW, Schurr A, Gozal D. Developmental differences in cortical and hippocampal vulnerability to intermittent hypoxia in the rat. Neurosci Lett 2001; 305: 197-201.

38 Row BW, Kheirandish L, Neville JJ, Gozal D. Impaired spatial learning and hyperactivity in developing rats exposed to intermittent hypoxia. Pediatr Res 2002; 52: 449-453.

39 Row BW, Liu R, Kheirandish L, Gozal D. Intermittent hypoxia is associated with oxidative stress and spatial learning deficits in the rat. Am J Respir Crit Care Med 2003; 167: 1548-1553.

40 Decker MJ, Hue GE, Caudle WM, Miller GW, Keating GL, Rye DB. Episodic neonatal hypoxia evokes executive dysfunction and regionally specific alterations in markers of dopamine signaling. Neuroscience 2003; 117: 417-425.

41 Row BW, Goldbart A, Gozal E, Gozal D. Spatial pre-training attenuates hippocampal impairments in rats exposed to intermittent hypoxia. Neurosci Lett 2003; 339: 67-71.

42 Scholle S, Scholle HC, Kemper A, et al. First night effect in children and adolescents undergoing polysomnography for sleep-disordered breathing. Clin Neurophysiol 2003; 114: 2138-2145. 